Research Paper

\title{
Driving Behavior Analysis of Elderly Drivers Passing Through Stop Sign Intersection Using Driving Simulator
}

\author{
Takashi Yonekawa $^{1)}$ Kazuya Sasaki $^{2)}$ Katsuhiko Iwazaki $^{3)}$ \\ 1)-3)TOYOTA MOTOR CORPORATION \\ 1200, Mishuku, Susono,Shizuka,410-1193, Japan (E-mail:takashi_yonekawa@mail.toyota.co.jp)
}

Received on February 19, 2014

Presented at the Second International Symposium on Future Active Safety Technology toward zero-traffic-accident on September 24, 2013

\begin{abstract}
In Japan it is well known that many crossing collisions occur at non-signalized intersections, especially elderly drivers are responsible for these accidents. Therefore, the traffic circumstance of stop sign intersections, where visibility is bad, was simulated by our highly realistic driving simulator. The visibility distance of the crossing vehicles and the confirmation action to pass through the intersection were evaluated with the time axis and the distance axis. The results found that the left/right safety checks of the elderly drivers were not sufficient. An ideal way of elderly driver's drive assist system was considered from the relation between elderly driver's driving action and accident factors based on these results.
\end{abstract}

KEY WORDS: human engineering, safety, older person, driver behavior, driving assessment, driving simulator [C2]

\section{Introduction}

Statistics show that the most common traffic accident pattern in Japan is rear-end collisions, followed by intersection collisions (Fig. 1) ${ }^{(1)}$. The statistics also show that intersection collisions involve a high proportion of elderly people. Consequently, studies have been made in real world driving conditions to evaluate the risks related to driving through intersections with a stop sign ${ }^{(2)}{ }^{(3)}$. However, variations in the traffic environment on ordinary public roads make it difficult to analyze driver behavior in these situations. Therefore, a highly realistic driving simulator (Fig. 2) ${ }^{(4)}$ was used to re-create the traffic environment at stop sign intersections with poor visibility. In this situation, the behavior of elderly drivers was measured and analyzed to identify the correlation between driving behavior and factors leading to accidents.

The simulated traffic environment featured stop sign intersections with intersecting road traffic, including bicycles. The visible range at the intersections and the actions of the driver to confirm safety to the left and right while passing through the intersections were evaluated on time and distance axes. This research then compared the driving behavior of elderly drivers aged 60 or over and drivers under 60 while passing through the intersections. It was found that elderly driver's confirmation actions of the left/right safety are insufficient. The relationship between the driving behavior of elderly drivers and accident factors was then applied to ideas for elderly driver support systems.

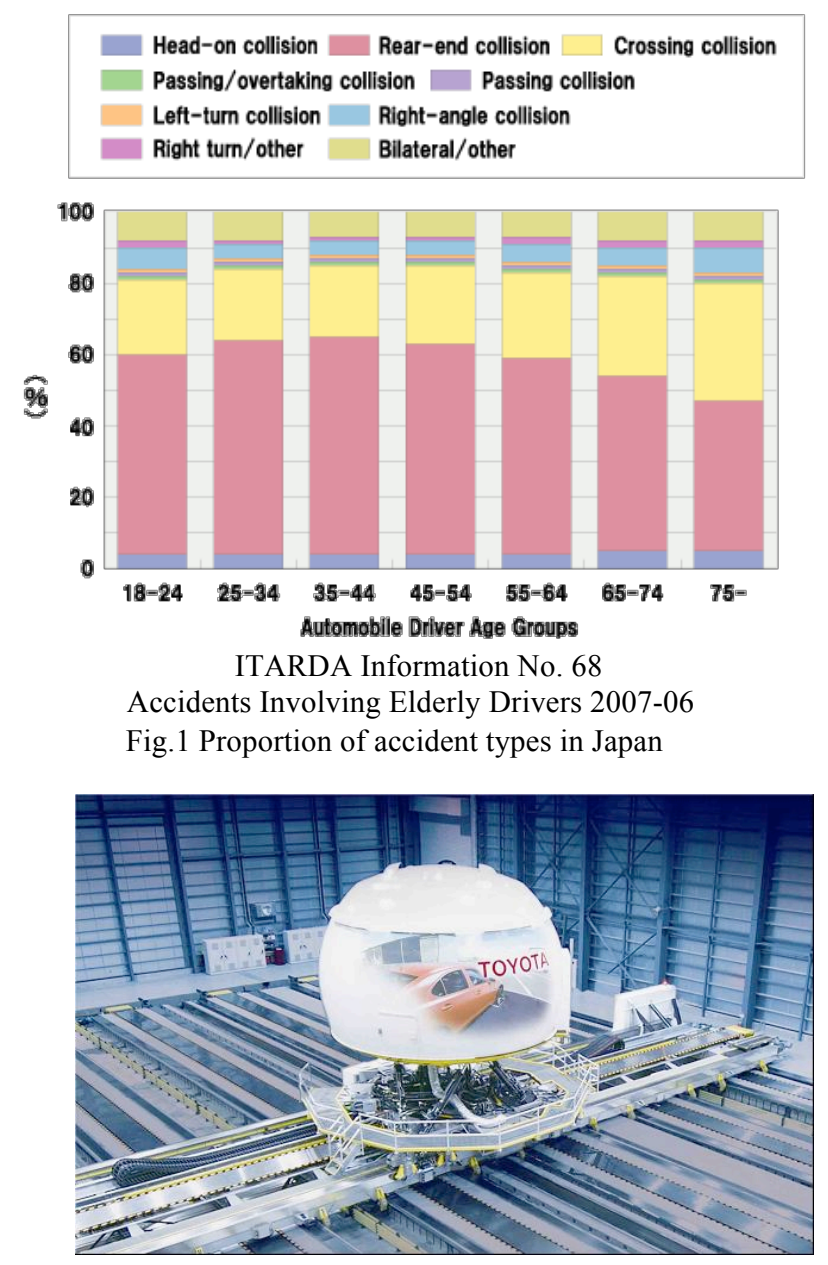

Fig.2 Highly realistic driving simulator 


\section{Test Method}

A highly realistic driving simulator (DS) was used to re-create the traffic environment at intersections. This is a high-performance DS that is capable of simulating the actual body sensation created by acceleration and deceleration, as well that created by left and right turns (Fig. 2). Two stop sign intersections were simulated in an urban driving course extracted from the real world. In this approximately 10-minute course, a single-lane approach to a stopsign intersection with poor visibility was repeated in two locations. As shown in Fig. 3, intersection 1 has a distance of 11 meters from the intersection stop line to a sidewalk that defines the crossing lane of a bicycle entering the intersection. Visibility at intersection 1 is limited by a building to the left and a parked truck to the right. Additionally, as shown in Fig. 4, intersection 2 has a distance from the intersection stop line to the sidewalk that defines the crossing lane of a bicycle entering the intersection of only 6 meters, shorter than that of intersection 1 . Visibility at intersection 2 is extremely poor due to a building on the left and the walls of railroad bridge support pillars on the right. Figure 5 compares the visible range of each intersection. In this figure, the $\mathrm{X}$-axis shows the distance in the driving direction with the stop line of intersection 2 as the zero-point. The Y-axis shows the visible ranges of the bicycle crossing lane and the vehicle crossing lanes on the left and right, considering uniform crossing lanes for both intersections. This figure indicates that there is little difference in the visible range between the crossing lanes of the bicycle coming from the left and the vehicle coming from the left for both intersections 1 and 2. In contrast, it also indicates that the visible range to the right at intersection 2 is extremely poor. At intersection 2, to confirm the location of a bicycle coming from the right at a distance of 20 meters, the front of the vehicle has to protrude across the crossing lane of the bicycle by 0.5 meters. However, at intersection 1, the location of a bicycle coming from the right at a distance of 20 meters can be confirmed from a position 4.5 meters before the crossing lane of the bicycle. Furthermore, a vehicle coming from the right at a distance of 40 meters can be confirmed from a position 7.5 meters before the crossing lane of the vehicle at intersection 1 , but only at a position 4.5 meters before the crossing lane of the vehicle at intersection 2 . This under lanes the large variation in visibility between the intersections. Fifteen test subjects aged 60 or over and eight test subjects under 60 drove over this simulated test course Intersection driving behavior was obtained for a total of 54 cases.

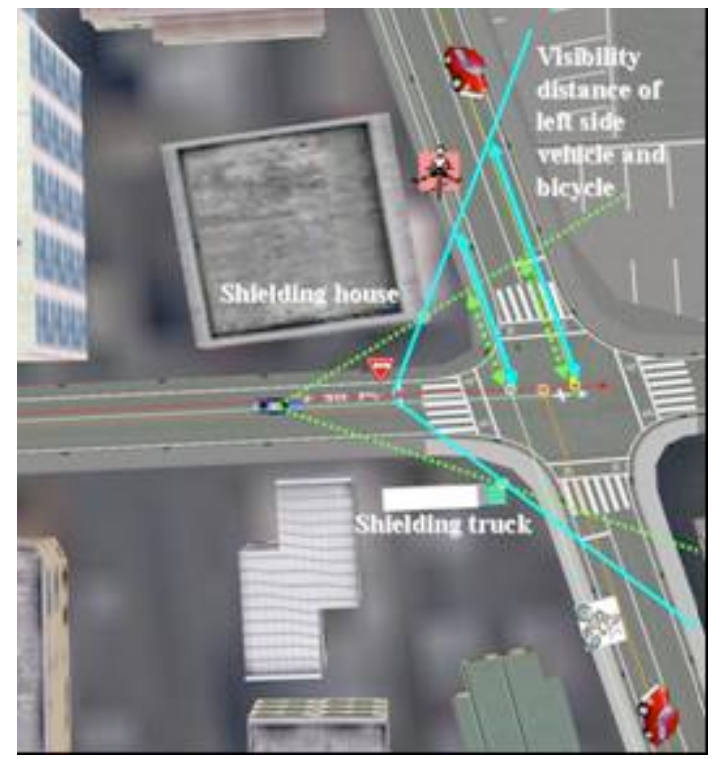

Fig.3 Layout of stop sign intersection 1

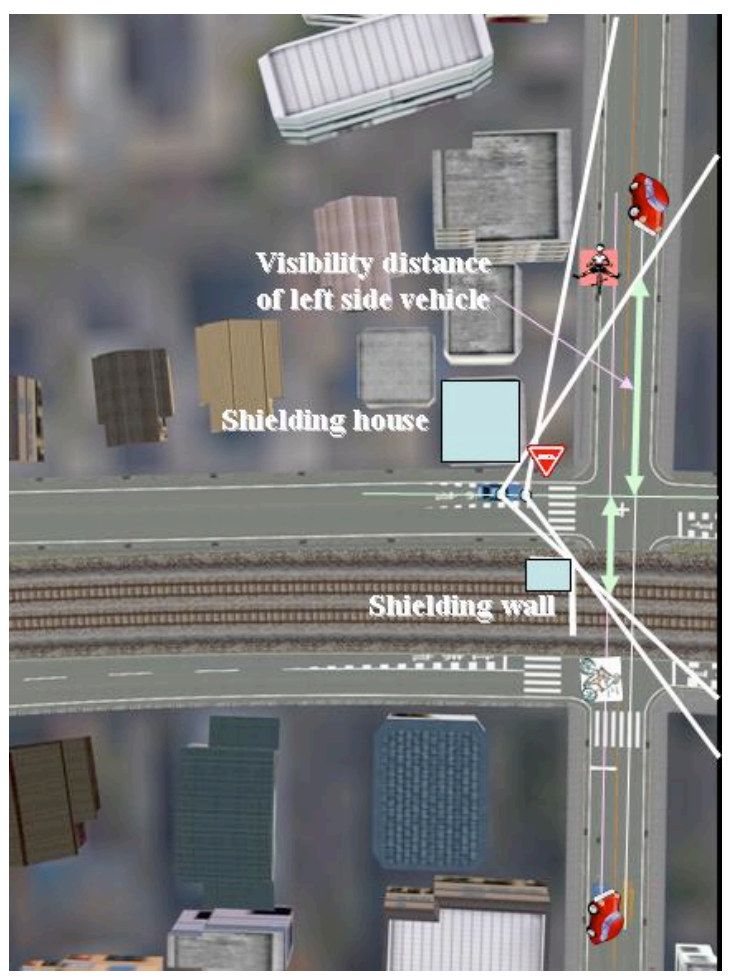

Fig.4 Layout of Stop sign intersection 2

Figure 6 is showing the detailed driving behavior when passing through Intersection 1. The graphs show the visible range of the crossing objects in respect to the distance from the stop line and time on the $\mathrm{X}$-axes. The bicycle and vehicle crossing lane slopes show the road crossing angle, because in this particular intersection the geometrical road crossing angle was about 76.4 deg. The driving behavior types included in these graphs include deceleration and slow moving of the driver's vehicle, left and right safety checks of the driver, the position of the driver's foot in preparation for braking, and so on. The relationship between visible range and safe driving behavior when passing through an intersection was analyzed based on this data.

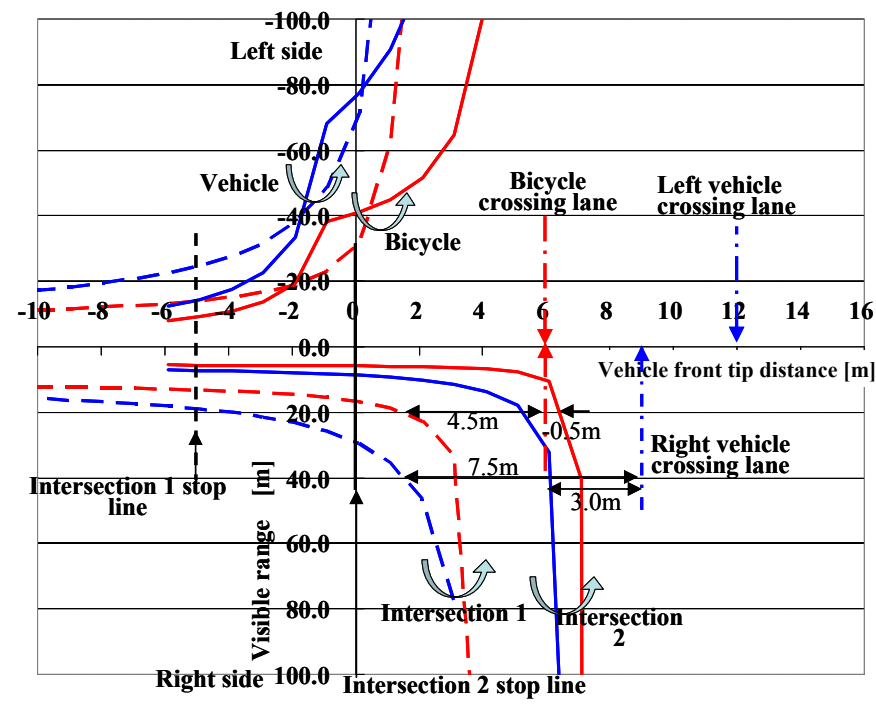

Fig.5 Visible range when approaching simulated intersections 

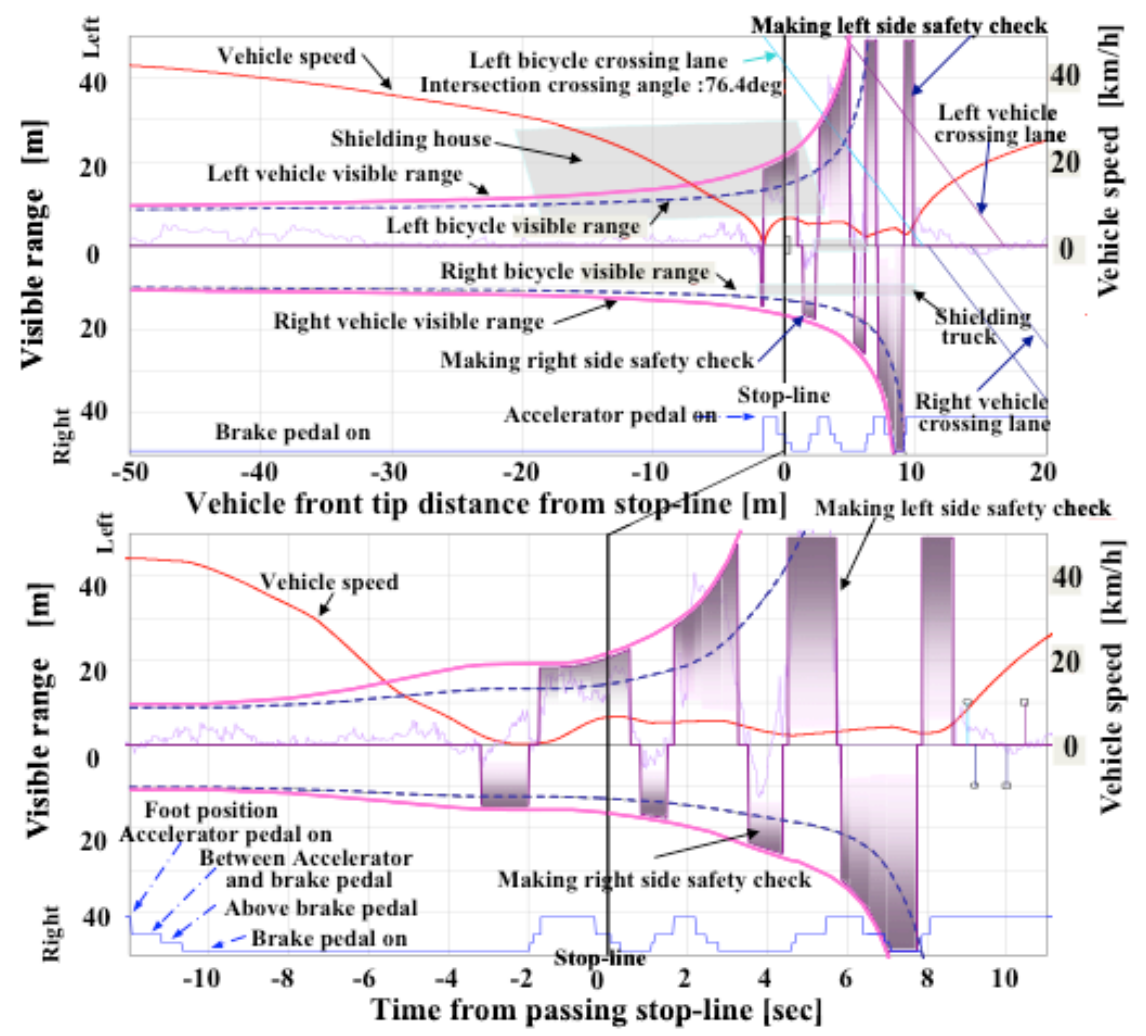

Fig.6 Example of driving activity profile at intersection 1

(vehicle speed, foot position for braking, making left and right side safety checks, visible range)

\section{Stopping Behavior Analysis}

The driver stopping behavior at the stop sign intersections was analyzed. Figure 7 shows the results for the frequency of stopping or decreasing speed. At intersection 2, which has extremely poor visibility, drivers stopped or decreased speed more frequently than in intersection 1 (more than once per crossing). In this analysis, a vehicle speed of less than $0.1 \mathrm{~km} / \mathrm{h}$ is defined as a stop and a speed of less than $3 \mathrm{~km} / \mathrm{h}$ is defined as a slow speed. At intersection 1, which has relatively good visibility, drivers stopped more frequently. Unlike the high frequency of cases in the real world in which drivers ignore the stop sign altogether, in this study the test subjects stopped carefully at the stop line. However, some drivers drove through intersection 1 without slowing down. The test subjects drove safer than in Kosaka's report ${ }^{(6)}$ which represents driving behavior at stop sign intersection in the real world. This is probably due to the fact that the drivers were aware that the DS experiment was being observed, and our subjects were safer drivers compare with Kosaka's report drivers. In the same way, Fig. 8 shows no difference in the first stopping position in respect to the stop line, regardless of the visible range at the intersection. The test subjects tended to stop before the stop line, which indicates that the drivers were using the line as a target to stop the vehicle, because the drivers were stopping same at the position of intersection 1 and 2. These behaviors, in which the drivers stopped before the stop line, suggest that the actual body sensation of deceleration in the DS may be insufficient. In the real world, many drivers stop passed the stop line in Kosaka's report. ${ }^{(6)}$ The quantitative stop position from the stop line on DS was different than real world. But in the safety stop maneuver at the intersection the stop line was used as a target to stop the vehicle. In contrast, Fig. 9 shows that the positions for stopping to reconfirm safety and to perform safety check behavior occurred after entering the intersection. At both intersections 1 and 2, the drivers stopped or slowed speed at position targeting the bicycle crossing lane, in which the sidewalks located differently 11 and 6 meters from the stop line, to confirm safety check. In addition, at poor-visibility intersection 2, drivers also stopped to confirm safety or slowed speed down even after entering the intersection. Stop : Speed $<0.1 \mathrm{~km} / \mathrm{h}$
Speed down $:$ Speed $<3 \mathrm{~km} / \mathrm{h}$

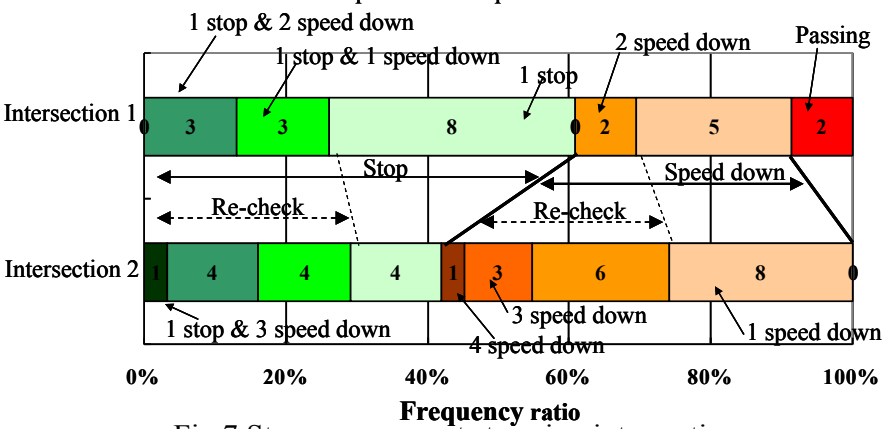

Fig.7 Stop maneuver at stop sign intersections

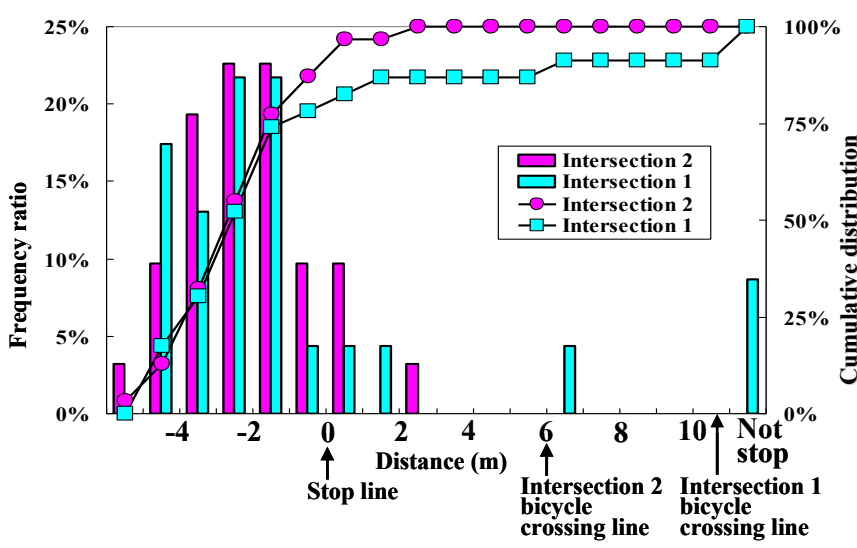

Fig. 8 Distance of first stop or lowest speed position at intersection 


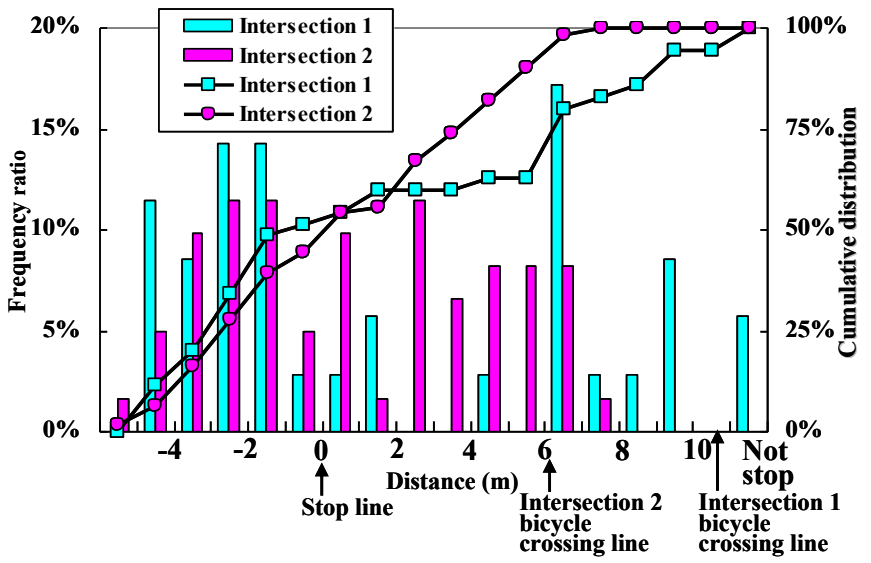

Fig.9 Distance of all stop or lowest speed positions from stop line on intersections

The speed of entering a stop sign intersection and the braking timing were calculated based on the distance from the stop line. The results are shown in Fig. 10. There was little difference in the stopping behavior of elderly drivers aged 60 or over and drivers under 60. Both entered the intersection at a speed that would allow the driver to stop the vehicle, while braking the vehicle at deceleration of approximately $1.0 \mathrm{~m} / \mathrm{s}^{2}$. However, a small number of the elderly drivers aged 60 or over overlooked the stop line and braked too late. Some also crossed the stop line without braking. This suggests that some kind of driver support that provides stop sign information or encourages the driver to stop would be effective as a countermeasure against elderly drivers failing to notice a stop sign intersection.

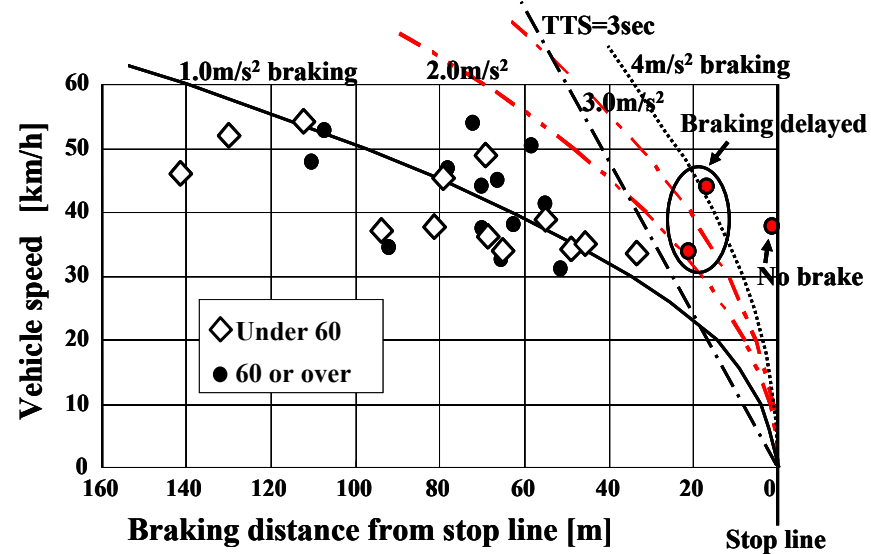

Fig.10 Relationship of braking distance from stop line with average deceleration and time to stop line at intersection 1 and 2

\section{Left and Right Side Safety Check Behavior Analysis}

At a stop sign intersection, the purpose of stopping the vehicle is to provide a time margin to check for other vehicles crossing the intersection and to avoid collision with vehicles entering the intersection at the same time. For this reason, the left/right safety check behavior of the drivers was analyzed. Figure 11 compares the vehicle speed between the stop line and entering the intersection (i.e., the bicycle crossing lane positions) at intersections 1 and 2, which have different visible ranges due to the arrangement of shielding objects. The results show that vehicle speed was lower at poor-visibility intersection 2 to give the driver more time to check for crossing objects. The behavior of elderly drivers aged 60 or over and drivers under 60 were also compared in the same way (Fig. 12). These results found that the drivers aged 60 or over drove faster over this region. In addition, Fig. 13 shows that $32 \%$ of drivers aged 60 or over reached the intersection entrance from the stop line in less than two seconds for both intersections 1 and 2 . This indicates that these drivers are not checking sufficiently for crossing objects. An analysis of the number of left/right safety checks found that $51 \%$ of drivers aged 60 or over confirmed safety in the left and right directions only once, confirmed safety only in the right or left direction, or did not check for safety at all, compared to only $26 \%$ of drivers under 60 (Fig. 14). This also indicates that these drivers are not checking sufficiently for crossing objects. Therefore, considering the reduction in cognitive performance of elderly drivers aged 60 or over, the risk of collision is extremely high for stop sign intersections.

An effective countermeasure against the delayed driving reactions of elderly drivers at an intersection is a readiness to brake to avoid potential collisions with crossing objects. The braking timing and foot position for braking are critically important when passing through an intersection. Therefore, this analysis also examined the braking timing and duration of foot position for braking when passing through the intersections (Fig. 15). The results found that $35 \%$ of drivers aged 60 or over did not prepare to press the brake in the intersections. In conclusion, the driving behavior of elderly people at a stop sign intersection suggests a connection between this behavior (i.e., insufficient safety checks of crossing objects after the stop line and insufficient preparation for the possible existence of crossing objects) and the high frequency of intersection accidents in which elderly drivers ignore a red traffic signal. Therefore, a support system that encourages the driver to stop is unlikely to help prevent intersection accidents involving elderly people by itself. It should be supplemented with a support system that confirms the existence of crossing objects and encourages the driver to prepare avoiding action or warns the driver if a vehicle is present.

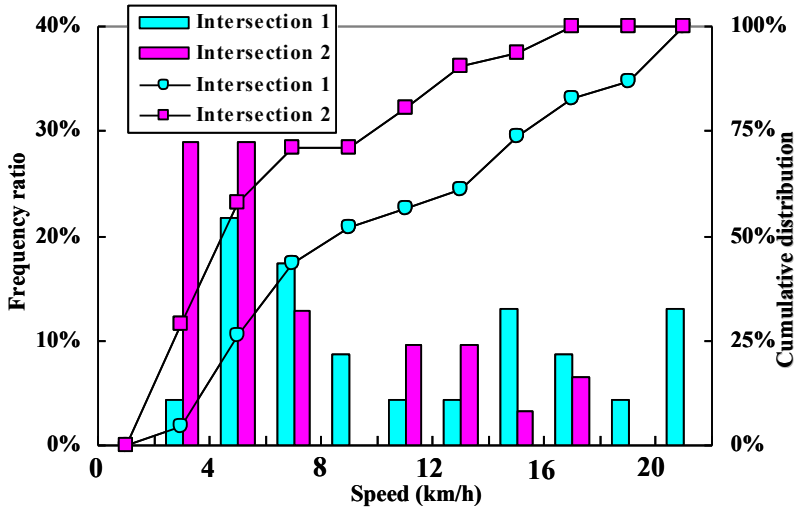

Fig.11 Average speed from stop line to entrance of intersection

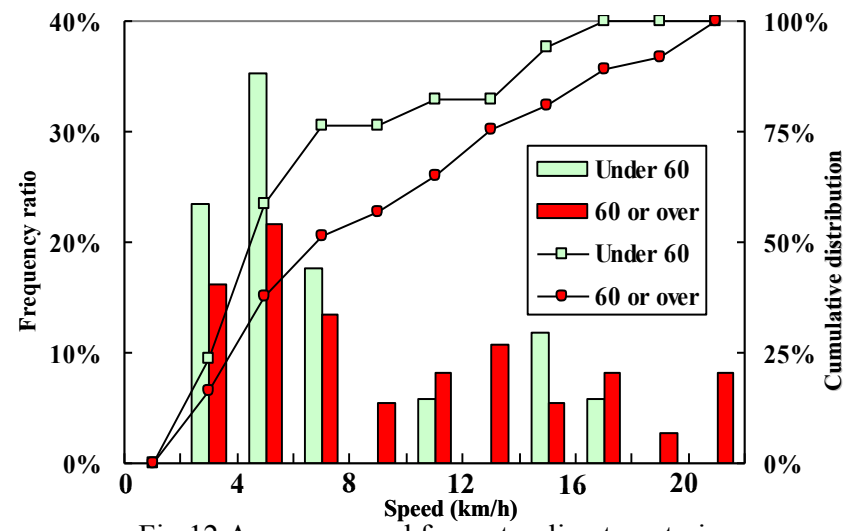

Fig.12 Average speed from stop line to entering intersection 1 and 2 


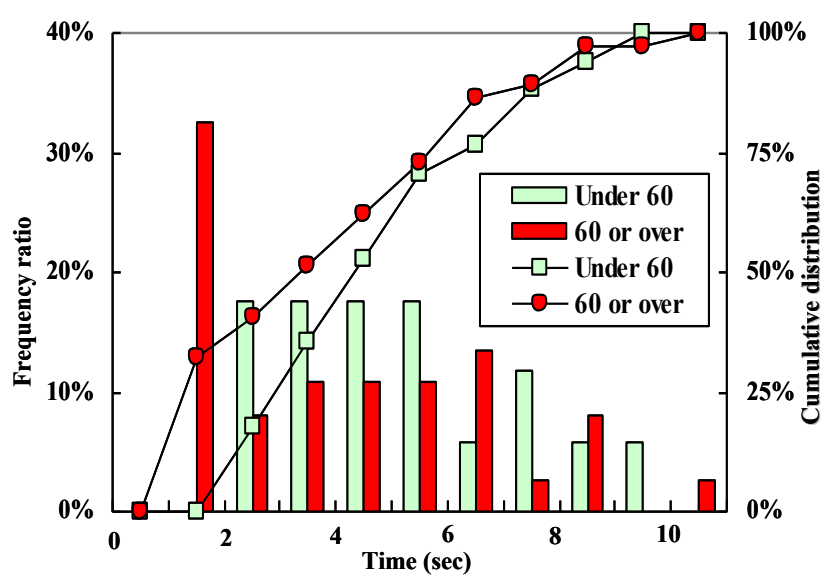

Fig.13 Time from stop line to entrance of intersection 1 and 2

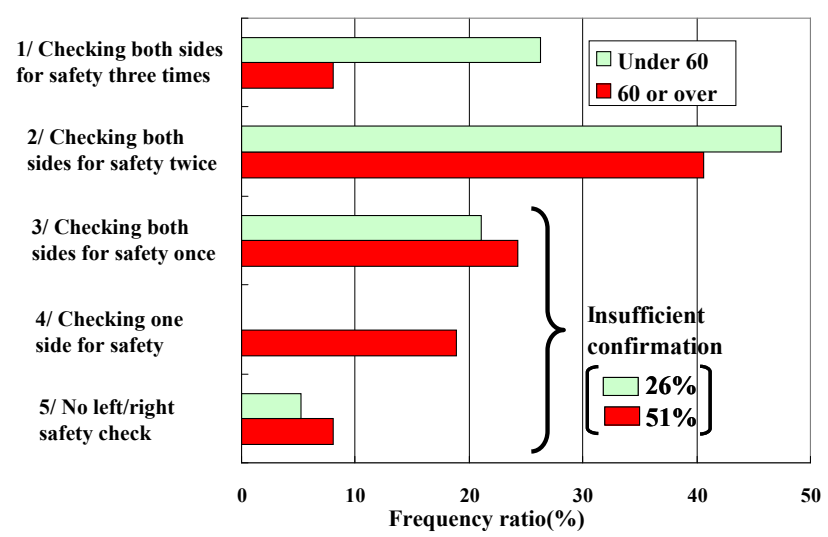

Fig.14 Frequency ratio of confirmation for crossing objects at intersection 1 and 2

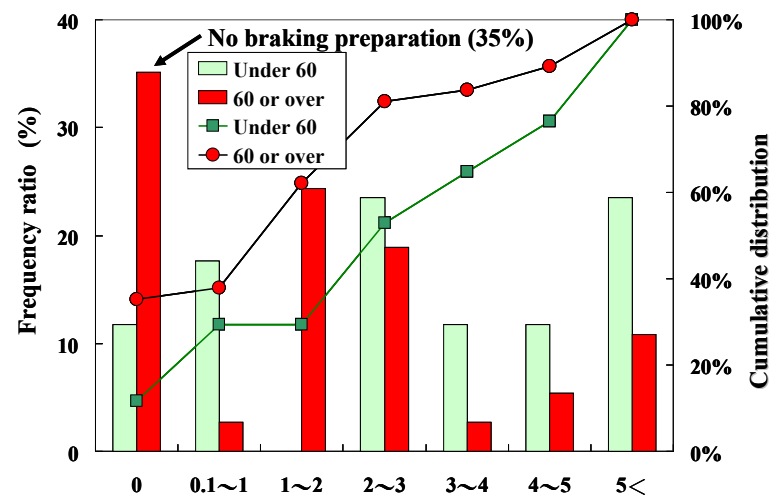

Braking preparation time when passing through intersection (sec)

Fig. 15 Frequency ratio of braking preparation times for crossing objects at intersection 1 and 2

\section{Time Margin to Collision of Crossing Objects}

The results described above confirmed that, compared to drivers under 60 , elderly drivers aged 60 or over do not carry out sufficient checks to the left and right for crossing objects at a stop sign intersection. However, since it is difficult to identify the degree of risk of this behavior, a risk evaluation method was developed for stop sign intersections. An existing method for evaluating the risk of collision at a stop sign intersection defines a larger collision avoidance margin based on the visible range and safety confirmation behavior of drivers as the crossing vehicle speed increases. This is because the driver is more likely to predict the existence of crossing vehicles travelling at these speeds ${ }^{(2)}$. Based on the same approach, another proposed evaluation method sees a lessening risk of collision as pedestrian walking speeds increase since the driver is more likely to predict the possibility of a pedestrian suddenly rushing out in front of the vehicle $^{(5)}$.

The crossing speed at which a driver can avoid an accident (i.e., the predicted crossing speed to avoid an accident) is theoretically a correct way of evaluating risk. However, it is difficult to use as an index of driving behavior risk for proposing ways of improving driver behavior to avoid accidents. Therefore a method of evaluating the risk of an intersection collision was developed by calculating the time margin for evasive action based on average driving behavior and the timing of left/right safety checks for crossing objects at each crossing speed. The calculation method is described below.

Figure 16 was created using the same method as the graph for detailed driving behavior when passing through the intersections described in the DS test method section above (Fig. 6). This data was then used to calculate a possible stopping speed curve. The stopping speed curves for the driver's foot position (which corresponds to the free running time) for each crossing objects were calculated using Equation (1) based on the distance from the stop position. These curves were defined as $\mathrm{Vb}(\mathrm{d})$ for a bicycle, $\operatorname{Vvr}(d)$ for a vehicle coming from the right, and $\operatorname{Vvl}(d)$ for a vehicle coming from the left. However, the free running time is assumed to be 0.3 seconds when the driver's foot is placed on the brake and 0.8 seconds when placed on the accelerator. Deceleration due to braking was assumed to be $5.0 \mathrm{~m} / \mathrm{s} 2$.

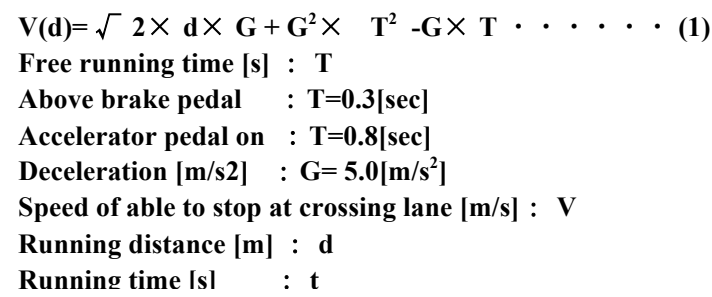

The distance-based stop speed curves calculated in Fig. 16 were converted to a time axis and plotted in Fig. 17. Based on the timing of intersection with the crossing object, the first timing that satisfies the (driver' $\mathrm{s}$ vehicle speed) $\leq$ (foot position stop curve) relationship in the direction of the stop line (Tbd: bicycle, Tdvr: vehicle coming from right, and Tdvl: vehicle coming from left) was set as the timing of the limit position at which the driver is capable of stopping.

Figure 17 was used to calculate the final timing that the driver faced the object before the limit position at which the driver is capable of stopping (Tbd, Tdvr, and Tdvl). This effective final confirmation timing is defined as Tdtbr for a bicycle coming from the right, Tdtbl for a bicycle coming from the left, Tdtvr for a vehicle coming from the right, and Tdtvl for a vehicle coming from the left. The visible range of the crossing object at this final confirmation target was then calculated (Dbr: bicycle coming from the right, Dbl: bicycle coming from the left, Dvr: vehicle coming from the right, and Dvl: vehicle coming from the left). However, 50 meters was used as the visible range of all objects over 50 meters.

Based on the final confirmation timing (Tdtbr, Tdtbl, Tdtvr, and Tdtvl), Equations (2) and (3) were used to calculate the time to intersection (Tcbr, Tcbl, Tcvr, and Tcvl) (i.e., the duration from the final confirmation timing to the intersection timing). It was assumed that the intersecting objects were travelling at a constant speed. The assumed crossing speeds of the bicycle and the driver's vehicle were $\mathrm{Vb}=20 \mathrm{~km} / \mathrm{h}$ and $\mathrm{Vv}=60 \mathrm{~km} / \mathrm{h}$, respectively.

Finally, the time margin for collision with a crossing object (Tmbr, Tmbl, Tmvr, and Tmvl) was calculated from the distance 
between the intersection passing time (Ttb, Ttvr, and Ttvl) of the driver's vehicle and the crossing object based on the final confirmation timing (Tdtbr, Tdtbl, Tdtvr, and Tdtvl) and the time to intersection (Tcbr, Tcbl, Tcvr, and Tcvl).
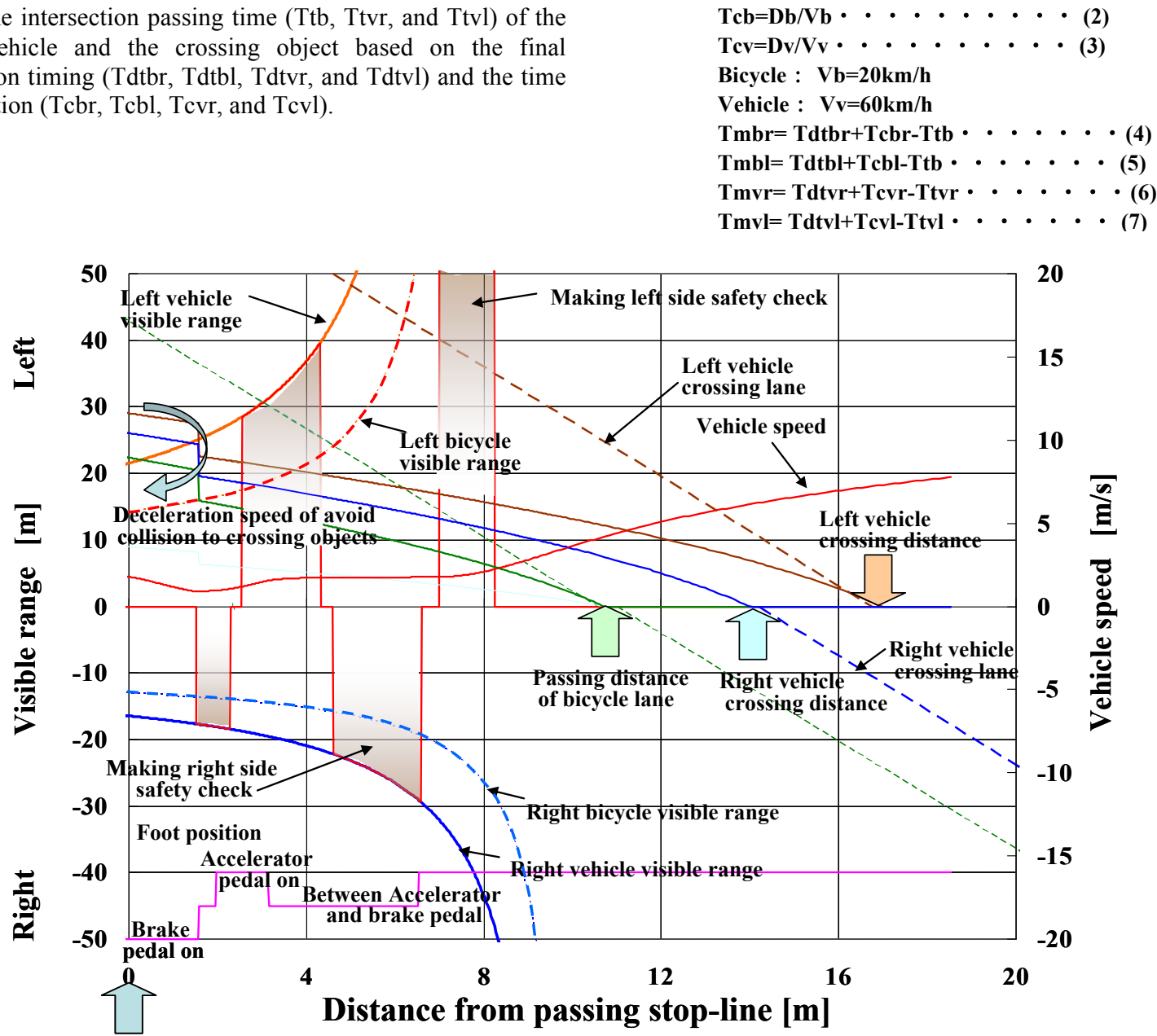

\section{Stop-line}

Fig.16 Calculation of deceleration speed to avoid collision with crossing object at intersection 1

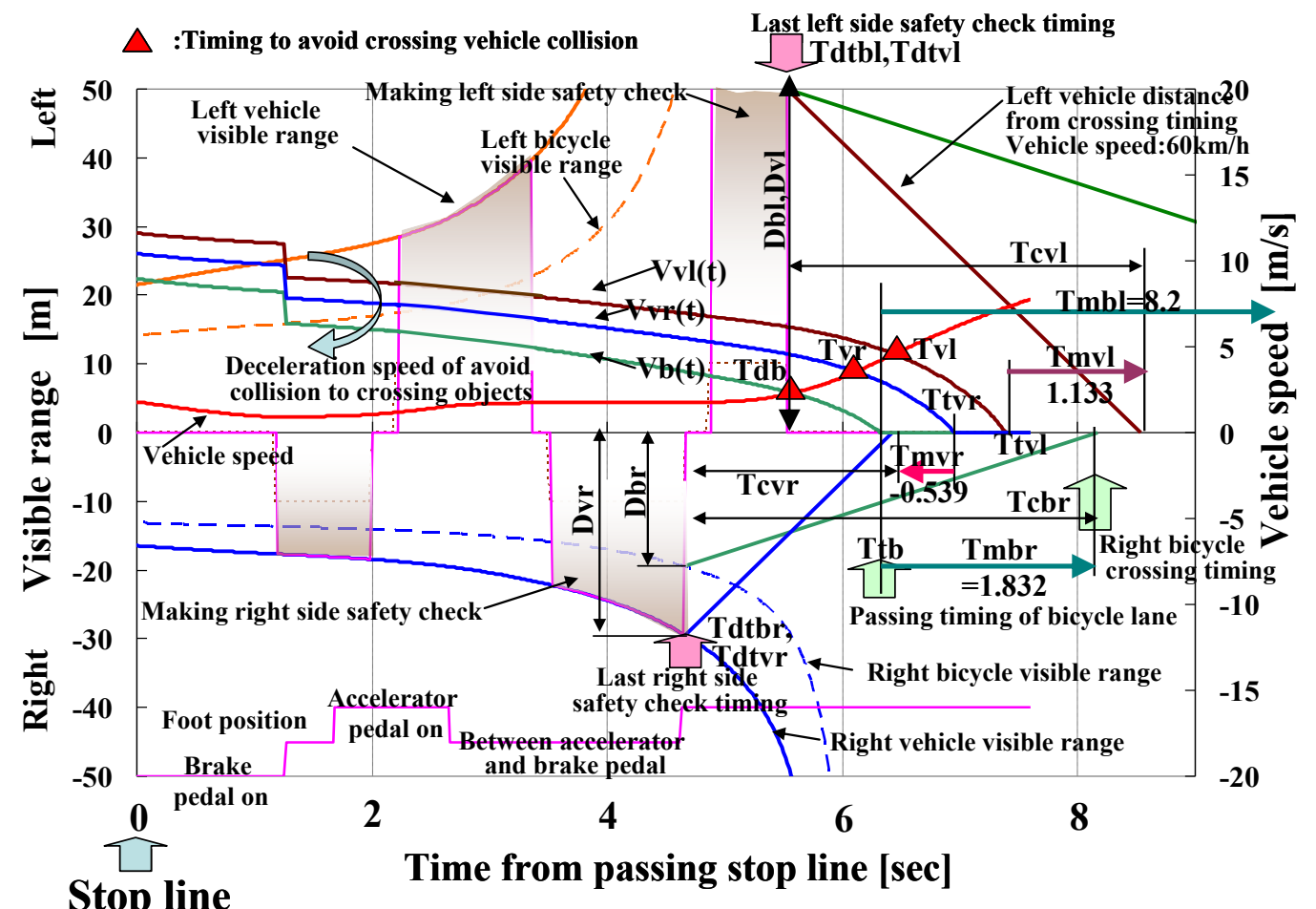

Fig.17 Calculation method of time margin to collision with crossing object at intersection 1 
This time margin for collision with a crossing object can be considered as follows. For example, since the time margin for a vehicle coming from the right in Fig. 17 is negative (Tmvr $=-0.559$ seconds), there is a high possibility of a collision with a vehicle coming from the right at a speed of $60 \mathrm{~km} / \mathrm{h}$. Therefore, a collision with a vehicle coming from the right at a speed of 60 $\mathrm{km} / \mathrm{h}$ can be avoided by creating a time margin of approximately 0.6 seconds. This is possible by reducing the speed through the intersection, increasing the stopping duration, or increasing the number of safety checks. In addition, since the time margin for collision with a bicycle coming from the right at $20 \mathrm{~km} / \mathrm{h}$ is relatively higher $(\mathrm{Tmbr}=1.832 \mathrm{~seconds})$, the driver has a reaction margin of 1.8 seconds in addition to the 0.8 seconds required to switch from pressing the accelerator to pressing the brake. Therefore, the collision risk can be evaluated in terms of the driver's reaction margin.

\section{Relationship between Time Margin and Intersection Visibility}

The time margin for collision with crossing objects was compared based on the differences in visible ranges at the intersections. Figure 18 shows the time margin for a vehicle coming from the right. When the time margin is negative, there is a risk of collision with a vehicle crossing at a speed of $60 \mathrm{~km} / \mathrm{h}$. The proportion of collision risk is higher at intersection $1(48 \%)$ than intersection $2(39 \%)$. This is because the time margin has a close distribution for intersection 1 since many drivers did not confirm safety at this intersection. In other words, drivers played close attention to safety checks at the intersection with poor visibility, creating a margin to avoid the vehicle coming from the right.

In contrast, Fig. 19 shows the risk of collision for a bicycle coming from the right at $20 \mathrm{~km} / \mathrm{h}$. In this case, the proportion of collision risk is higher at poor-visibility intersection $2(42 \%)$ than intersection $1(26 \%)$, which indicates a shorter time margin. In other words, the driver's safety checks are insufficient for a bicycle coming from the right at poor-visibility intersection 2

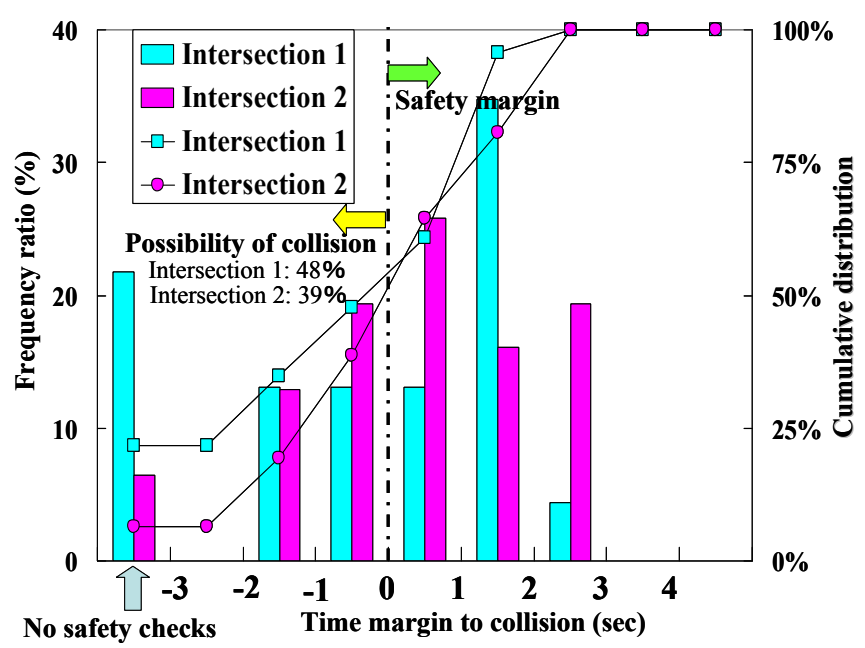

Fig.18 Time margin for collision with vehicle coming from right in accordance with difference in visible range

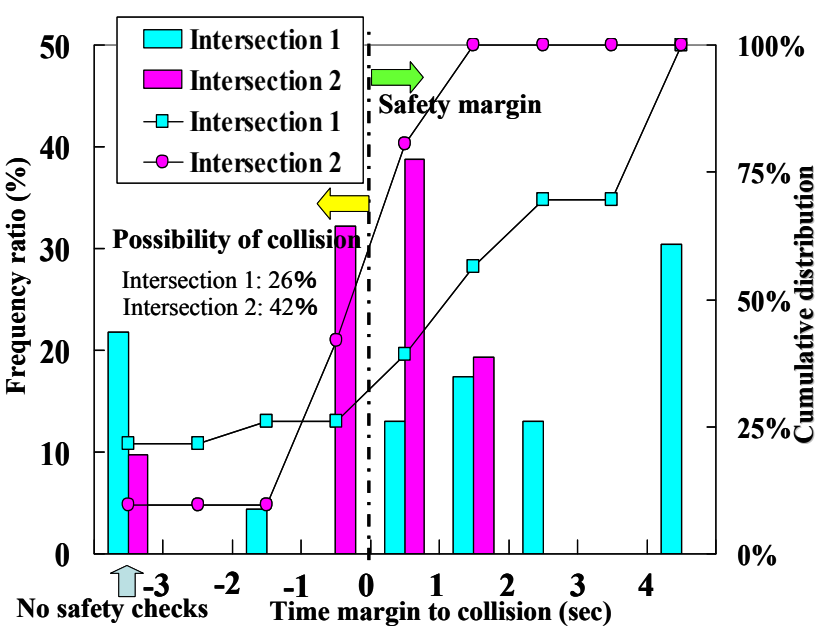

Fig.19 Time margin for collision with bicycle coming from right in accordance with difference in visible range

\section{Relationship between Time Margin and Driver Age}

The time margin for collision with crossing objects was also compared based on the age of the driver (60 or over and under 60 ). The proportions of collision risk shown in Fig. 20 (vehicle coming from right) and Fig. 21 (bicycle coming from left) show that roughly $20 \%$ of drivers aged 60 or over made no safety checks to the right, the direction with poor visibility. Consequently, the collision risk of drivers aged 60 or over for vehicles coming from the right was between $40 \%$ and $50 \%$ compared to only approximately $20 \%$ for drivers under 60 . Therefore, elderly drivers have twice the risk of colliding with an object at an intersection with poor visibility.

Figure 22 shows the collision risk with a vehicle coming from the left. Although drivers under 60 checked for safety while allowing a certain time margin, $22 \%$ of drivers aged 60 or over ran the risk of collision with a vehicle coming from the left at a speed of $60 \mathrm{~km} / \mathrm{h}$. However, only $25 \%$ of drivers had a time margin for collision of less than 1 second. In other words, although the accident risk is lower at intersections with good visibility, elderly drivers are three times more likely to have an accident than drivers under 60 .

This research defined collision avoidance behavior and the speed of crossing objects. It then calculated the time margin for collision with crossing objects based on the visible range and left/right safety checks when passing through the intersection. As a result, this research determined quantitatively that the safety checks of drivers aged 60 or over are insufficient at stop sign intersections. Consequently, a support system that encourages an elderly driver to stop is unlikely to help prevent intersection accidents by itself. Instead, a support system should be provided that encourages the driver to confirm safety to the left and right and helps the driver to avoid a collision with crossing objects. 


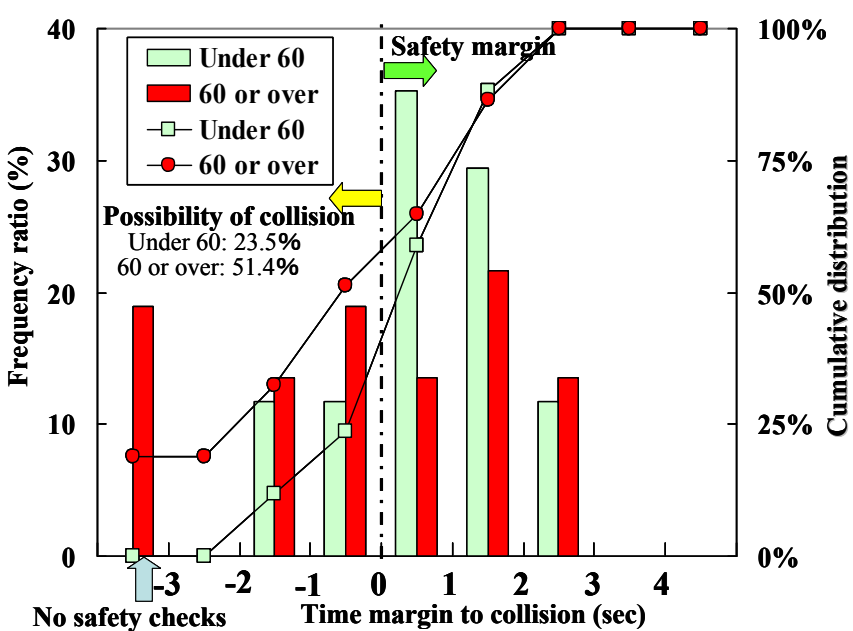

Fig.20 Time margin for collision with vehicle coming from right for elderly drivers at intersection 1 and 2

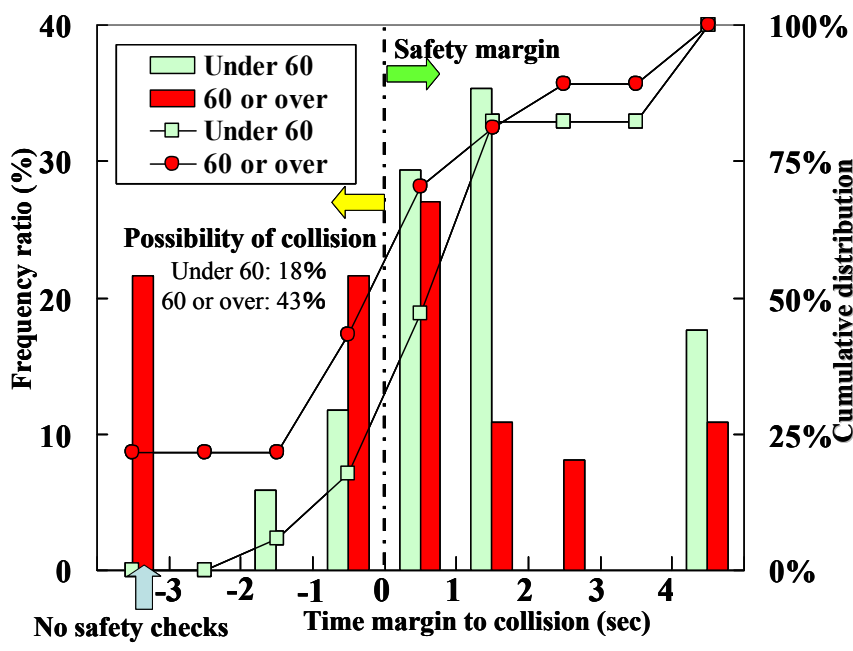

Fig.21 Time margin for collision with bicycle coming from right for elderly drivers at intersection 1 and 2

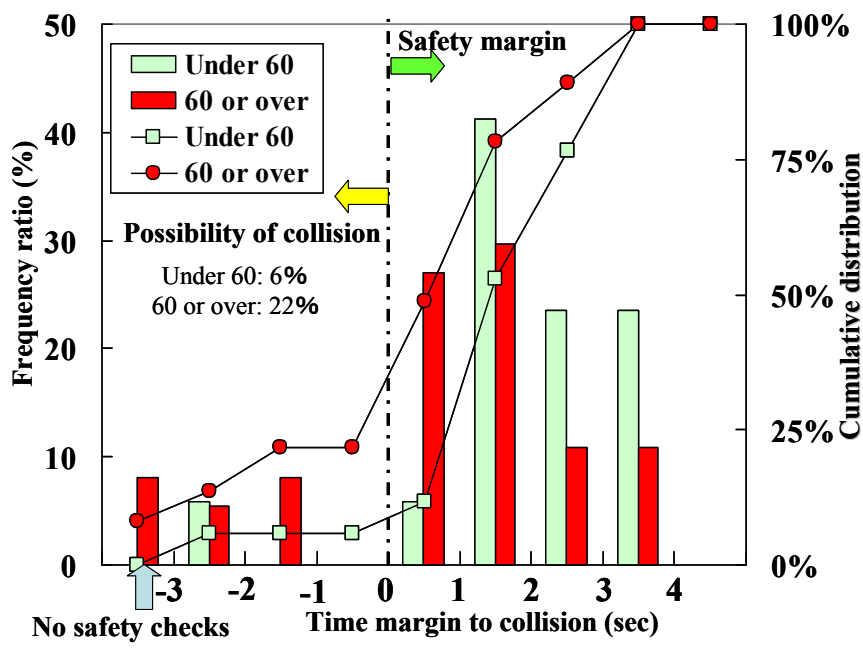

Fig.22 Time margin for collision with vehicle coming from left for elderly drivers at intersection 1 and 2

\section{Conclusion}

A driving simulator was used to evaluate the stopping and left/right safety check behavior of elderly drivers at a stop sign intersection. The following points were identified.

1. There were no major differences in the stopping behavior of elderly drivers aged 60 or over and drivers under 60 . However, the evaluation found that some elderly drivers aged 60 or over failed to stop or delayed the start of braking due to overlooking or delayed realization of the stop sign.

2. After stopping at the stop line, approximately $26 \%$ of drivers under 60 either failed to confirm to the left and right or performed insufficient safety checks (i.e., safety in both the left and right directions was confirmed less than twice) before accelerating through the intersection. However, the same phenomenon was observed in $51 \%$ of elderly drivers aged 60 or over, suggesting that elderly drivers do not confirm safety sufficiently.

3. This research defined collision avoidance behavior and the speed of crossing objects. It then calculated the time margin for collision with crossing objects based on the visible range and left/right safety checks when passing through the intersection. The results of this calculation determined quantitatively that the safety checks of drivers aged 60 or over are insufficient and that the accident risk of these drivers is twice as high as other drivers.

4. Elderly drivers require a support system that encourages the driver to confirm safety to the left and right and helps the driver to avoid a collision with crossing objects, rather than one that simply provides a warning to stop when the driver overlooks a stop sign.

\section{References}

(1)Institute for Traffic Accident Research and Data Analysis (ITARDA) : Accidents Involving Elderly Drivers 2007-06 : ITARDA Information No.68, (2007)

(2) Yusuke Kasuya, Shigeki Umehara, Masaru Noda, Hirokazu Nishitani, Kazuya Sasaki : Search for Model Behavior When Passing Through Non-signalized Intersection Based on Highest Admitted Speed (HAS) : (in Japanese with English summary), JSAE 20105110, 2010 JSAE Annual Spring Congress Proceedings, No.37-10, p.13-16, Tokyo, May 9, (2010)

(3) Tatsuya Kamon, Shigeki Umehara, Hiroaki Kosaka, Masaru Noda, Hirokazu Nishitani, Yoji Mizoguchi, Masumi Kobana, Kazuya Sasaki : Evaluating Risk Levels of Driver Behaviors on Basis of Vehicle Speed and Driver Eye-Movement and Pedal Operation : JSAE20104647, Review of Automotive Engineering, p.144-149(2010).

(4) Takahiko Murano, Takashi Yonekawa, Masami Aga, and Sueharu Nagiri : Development of High-Performance Driving Simulator: SAE 09AC-0133, SAE 2009 World Congress, Detroit, MI, (2009)

(5) Machiko Hiramatsu, Takashi Sunda, Motoki Shino, Minoru Kamata : A Study of a Method for Quantifying Drivers' Sensitivity to Potential Risks: (in Japanese with English summary), JSAE 20125588, JSAE 2012 Annual Autumn Congress Proceedings, No.85-12, p.1-4, Osaka, Oct. 3, (2012)

(6) Hiroaki Kosaka, Takuya Hashikawa, Masanori Takemoto, Hirokazu Nishitani, Masaaki Uechi, Kazuya Sasaki : Comparison of Car's Speed Passing through a Non-signalized Intersection without Right of Way among Different Intersection Environments : (in Japanese with English summary), JSAE 20085152, 2008 JSAE Annual Spring Congress Proceedings, No.1-08, p.13-16, Tokyo, May 21, (2008) 